\title{
Separation of Strangeness from Antistrangeness in the Phase Transition from Quark to Hadron Matter: Possible Formation of Strange Quark Matter in Heavy-Ion Collisions
}

\author{
Carsten Greiner, Peter Koch, and Horst Stöcker \\ Institut für Theoretische Physik, Johann Wolfgang Goethe Universität, D-6000 Frankfurt am Main, West Germany
}

(Received 10 November 1986; revised manuscript received 25 March 1987)

\begin{abstract}
We present a mechanism for the separation of strangeness from antistrangeness in the deconfinement transition. For a net strangeness of zero in the total system, the population of $s$ quarks is greatly enriched in the quark-gluon plasma, while the $\bar{s}$ quarks drift into the hadronic phase. This separation could result in "strangelet" formation, i.e., metastable blobs of strange-quark matter, which could serve as a unique signature for quark-gluon plasma formation in heavy-ion collisions.
\end{abstract}

PACS numbers: $25.70 . \mathrm{Np}, 12.38 . \mathrm{Mh}$

It has recently been proposed that quark matter with finite net strangeness might be a metastable ${ }^{1,2}$ or even an absolutely stable ${ }^{3,4}$ state of strongly interacting matter at finite chemical potential and zero temperature. Such "strangelets," i.e., blobs of multistrange quark matter, if detected, would most probably be the only form of quark matter not subject to rapid decay. ${ }^{3,4}$

On the other hand, it was also shown that equal amounts of strange and antistrange quarks are abundantly produced in a hot quark-gluon plasma (QGP), created, e.g., in the early Universe or in high-energy heavy-ion collisions. The strangeness saturates the phase space in a baryon-rich environment after a very short equilibration time, $t \simeq 10^{-22} \mathrm{sec}$, which may actually be shorter than the duration of a nuclear collision. ${ }^{5-7}$ Of course, in heavy-ion collisions the net strangeness of the QGP is zero from the onset. Up to date, therefore all model calculations (see, for example, the work of Lee, Rhoades-Brown, and $\mathrm{Heinz}^{8}$ ) for the confinement phase transition from the QGP into the gas of nonstrange, strange, and antistrange hadrons ( $\mathrm{HG}$ ) have assumed that the net strangeness is equal to zero in each phase separately.

However, in the following we demonstrate that during the phase transition there is a building up of a large antistrangeness content in the hadron matter while the quark-gluon plasma retains a large net strangeness excess-although the net strangeness of the combined QGP and $H G$ is equal to zero. This separation will occur only when the system carries a net positive (or negative) baryon number.

Hence we propose a viable mechanism for strangelet formation in initially nonstrange matter. Such nearly neutral objects would be most fascinating to study. Strangelets-if produced-would serve as a unique signature for the formation of a QGP.

The present approach relies on the assumption ${ }^{8-12}$ that the relaxation times for chemical transmutations as well as the hadronization time are small compared to the lifetime of the mixed phase in nuclear collisions. This assumption is supported by recent calculations. ${ }^{5-7}$ Accord- ing to these studies ${ }^{6,7}$ the duration of the phase transition is greatly enhanced by the conservation of the total entropy. $11,13,14$

Thus, entropy conservation is the first constraint for the transition process, i.e., $\sigma V=\sigma_{\mathrm{QGP}} V_{\mathrm{QGP}}+\sigma_{\text {had }} V_{\text {had }}$. Here $\sigma$ and $V$ are the entropy densities and volumes of the different phases. The total volume is given by $V=V_{\mathrm{QGP}}+V_{\text {had }}$.

The deconfinement phase transition between a large number of mesons and baryon resonances and nearly massless quarks and gluons is taken to be of first order. $^{9-12}$ We have assumed isospin symmetry, i.e., the chemical potential $\mu_{\text {up }}=\mu_{\text {down }}=\mu_{q}=\mu^{B} / 3$. The Gibbs equilibrium between the two phases is given by $T_{\mathrm{QGP}}$ $=T_{\text {had }}$ (thermal equilibrium), $P_{\mathrm{QGP}}=P_{\text {had }}$ (mechanical equilibrium), $\mu_{\mathrm{QGP}}^{B}=\mu_{\mathrm{had}}^{B}$ and $\mu_{\mathrm{QGP}}^{s}=\mu_{\mathrm{had}}^{s}$ (chemical equilibrium); $\mu_{s}$ is the chemical potential for strangeness, which controls the strangeness content. We emphasize the significance of the last condition: In a Gibbs phase equilibrium the chemical potentials are continuous across the phase boundary whereas the corresponding densities are discontinuous (this has been discarded in Ref. 8).

The requirement of total strangeness and baryon number conservation yields two additional constraints

$$
\begin{gathered}
S=V_{\mathrm{QGP}}\left(\rho_{s}-\rho_{\bar{s}}\right)+V_{\mathrm{had}}\left(\rho_{K}+\rho_{Y}-\rho_{\bar{K}}-\rho_{\bar{Y}}\right)=0 \\
\begin{aligned}
A=V_{\mathrm{QGP}} \frac{1}{3}\left(\rho_{q}-\rho_{\bar{q}}+\rho_{s}-\rho_{\bar{s}}\right) \\
\quad+V_{\mathrm{had}}\left(\rho_{N}-\rho_{\bar{N}}+\rho_{Y}-\rho_{\bar{Y}}\right) .
\end{aligned}
\end{gathered}
$$

Here the $\rho_{i}$ 's are the densities for the strange and nonstrange quarks $(i=s, \bar{s}, q, \bar{q})$, kaons $(i=K, \bar{K})$, hyperons $(i=Y, \bar{Y})$, and nucleons and their resonances $(i=N, \bar{N})$, which, in Boltzmann approximation for the strange particles, are given by

$$
\rho_{i}=g_{i}\left(T^{3} / 2 \pi^{2}\right)\left(m_{i} / T\right) K_{2}\left(m_{i} / T\right) \exp \left(\mu_{i} / T\right) .
$$

Only kaons and hyperons with strangeness $s= \pm 1$ are taken into account here. We estimate the effect of heavier strange hyperons, using Eq. (2), and find $n_{\Xi} / n_{Y}$ 
$\approx 0.05-0.1$ and $n_{\Omega} / n_{Y} \approx 0.005-0.001$ for $\mu_{q}, T$ in the hundred-megaelect ronvolt region.

The flavor composition of strange hadrons and quarks yields

$$
\begin{aligned}
& \mu_{K, \bar{K}}= \pm \mu_{q} \mp \mu_{s}, \\
& \mu_{Y, \bar{Y}}= \pm 2 \mu_{q} \pm \mu_{s}, \\
& \mu_{s, \bar{s}}= \pm \mu_{s} .
\end{aligned}
$$

Let us point out again that in the present approach the net strangeness in each separate phase need not vanish, although $S_{\text {total }}=0$. On the contrary, we emphasize that this would be inconsistent with the Gibbs equilibrium conditions.

The influence of the strange particles on the equation of state of both phases and on the location of the phase transition is small ${ }^{8}$ and is therefore neglected in the following. We employ the bag model for the quark-gluon plasma and use a thermal gas of hadrons for the confined matter. ${ }^{9-12}$ Interactions are neglected, i.e., $\alpha_{s}=0$. The phase transition curves of Refs. 11 and 12, which include a large number of nonstrange mesons and baryons, are employed to study the evolution of the strangeness in the present work.

Baryon number and entropy conservation force the system to expand along the critical curve $(T, \mu)$ by converting plasma volume into hadron gas volume. " For a given entropy and baryon number, the temperature and the baryochemical potential specify the volumes occupied by the two phases. ${ }^{11}$ The strange chemical potential $\mu_{s}$ needs to be specified in addition to ensure $S_{\text {total }}=0$.

Strangeness conservation leads to the following expression

$$
\mu_{s}=\frac{T}{2} \ln \frac{V_{\mathrm{QGP}} 3 W_{s} \exp \left(-\mu_{q} / T\right)+V_{\mathrm{had}}\left[W_{K}+\left(W_{\Lambda}+3 W_{\Sigma}\right) \exp \left(-3 \mu_{q} / T\right)\right]}{V_{\mathrm{QGP}} 3 W_{s} \exp \left(+\mu_{q} / T\right)+V_{\mathrm{had}}\left[W_{K}+\left(W_{\Lambda}+3 W_{\Sigma}\right) \exp \left(+3 \mu_{q} / T\right)\right]}+\mu_{q},
$$

where $W_{i}=\left(m_{i} / T\right)^{2} K_{2}\left(m_{i} / T\right)$. If the hadron phase is eliminated $\left(V_{\text {had }}=0\right)$ the well-known result ${ }^{5} \mu_{s}=0$ is recovered: $s$ and $\bar{s}$ quarks are produced in pairs only. On the other hand, for vanishing QGP $\left(V_{\mathrm{QGP}}=0\right)$ zero net strangeness leads to a nonzero value of the strange chemical potential, ${ }^{5} \mu_{s} \neq 0$ : Different strange-particle production modes show up in the dominance of the associated production over the direct pair production at finite baryon density.

During the coexistence of the QGP and HG, an additional channel opens up for the strangeness: Besides the associated production and pair production in the hadron gas it is possible to have, for example, associated production of a $K$ meson in the sector of the hadron phase and the $s$ quark staying in the QGP. Consequently, the strange quarks in the plasma phase do acquire a chemical potential $\mu_{s}$ different from 0 . This leads to a ratio of $s$ to $\bar{s}$ quarks larger than 1 in the QGP and to a diminished hyperon abundance in the hadronic sector. This phenomena only occurs for finite net-baryon densities, $\rho_{B}, \mu_{B} \neq 0$. For $\mu_{q}=0$, i.e., vanishing net-baryon density, the transition is also completely symmetric for strange particles and antiparticles, i.e., $\mu_{s}=0$ and $s / \bar{s}=1$. This is shown in Fig. 1 for temperatures and baryochemical potentials along the phase boundary of Refs. 11 and 12 with use of different values of the bag constant and of strange-quark mass.

Observe that in Fig. 1(a), where the original MIT bag-model constant is used, $s / \bar{s}$ exceeds 10 for $\mu_{B}=150$ $\mathrm{MeV}$. Figure 1(b) shows the analogous result obtained with a larger bag constant, $B^{1 / 4}=210 \mathrm{MeV}$. Note that the ratio does not exceed 3 for this parameter set. Also shown is the path that the system must take in an isentropic expansion with $\sigma / A=10$. We have used the isentropic expansion scenario ${ }^{11}$ based on the simple onedimensional model of Bjorken ${ }^{15}$ to simulate the dynam- ics of the transition: This treatment makes use of the assumption that the expanding quark-gluon plasma undergoes a phase transition at constant entropy and baryon number and, consequently, constant $\sigma / A$. Thus we get an impression of the time dependence of the process discussed above. Figure 1(c) shows the fraction of strange quarks to all quarks present in the QGP as a function of $\mu_{q}$. The same bag-model parameters as in Fig. 1(b) are used. Note that $f_{s}$ can exceed 0.5. The path of an isentropic expansion with $\sigma / A$ is also demonstrated. Figure 2 shows the time dependence of the $s / \bar{s}$ ratio and of the fraction of strangeness for the isentropic expansion scenario shown in the previous figures. Of special importance is the fact that the accumulation of $s$ quarks in the plasma phase grows with decreasing plasma volume (the fraction $\kappa$, introduced in these figures, denotes the fraction the hadron gas volume occupies compared to the total volume). This opens up the possibility that $s$ quarks may be bound not only in hyperons and strange mesons: They could form strange quark clusters which might be metastable objects.

For small temperatures and large $\mu_{q}$, the ratio $\rho_{s} / \rho_{\bar{s}}$ becomes very large even for small $\kappa$, although the net strangeness fraction given by $f_{s}=\left(\rho_{s}-\rho_{\bar{s}}\right) / \rho_{B, \mathrm{QGP}}$ becomes very small. The ratio $f_{s}$ gives the net strangequark concentration in the separating quark droplets. For $\kappa=1$ values of 0.85 for $f_{s}$ can be reached, i.e., about one strange quark per baryon number. Rapid expansion can therefore result in the formation of droplets of strange-quark matter, which might be metastable.

The possibility of separating strange quarks from antistrange quarks in the QGP-HG transition can lead - towards the late stage of the phase transition- to a tremendous enrichment of strange quarks in the QGP.

A fully dynamical scenario of the phase transition 


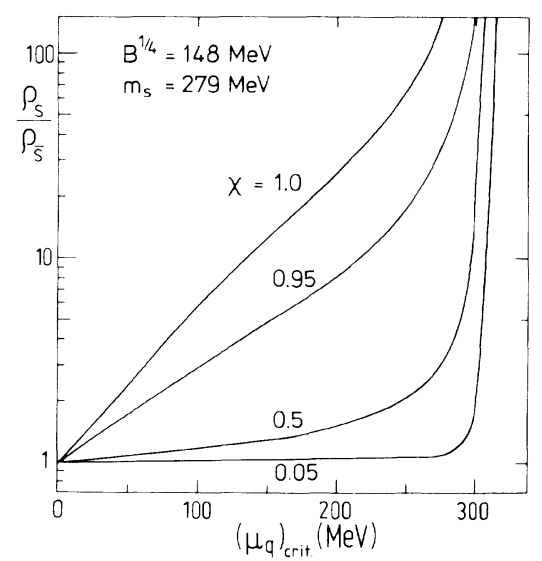

(a)

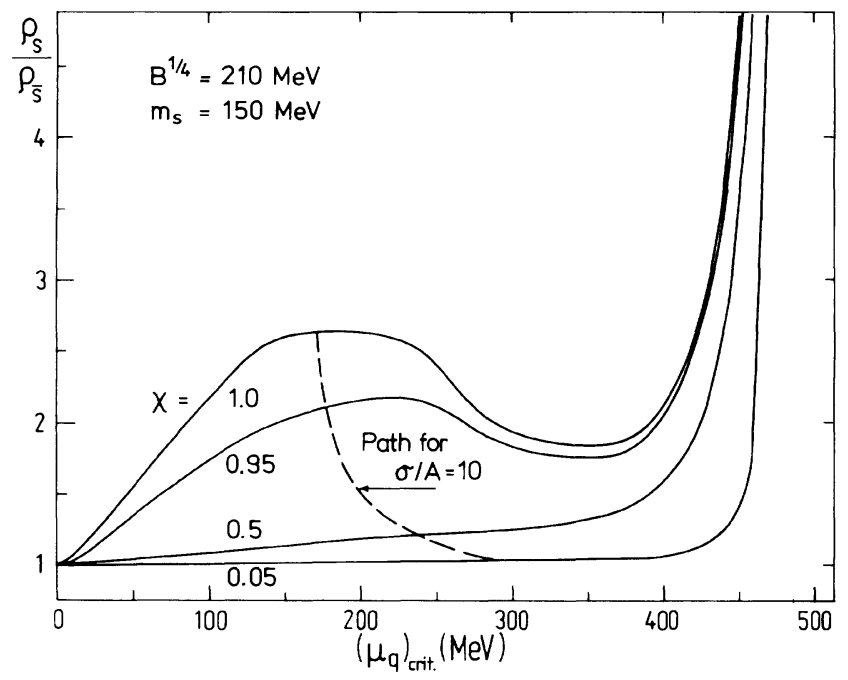

(b)

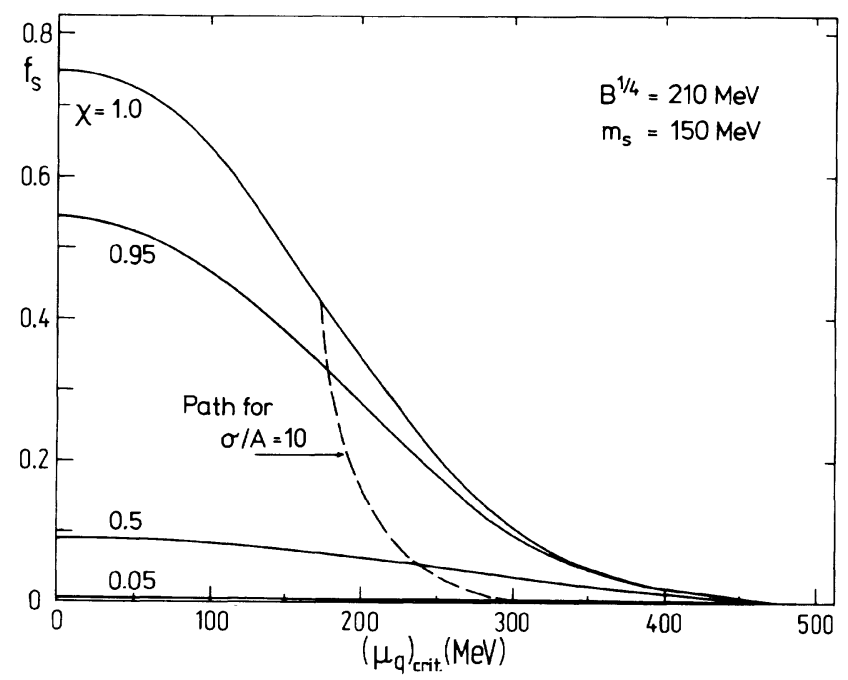

(c)

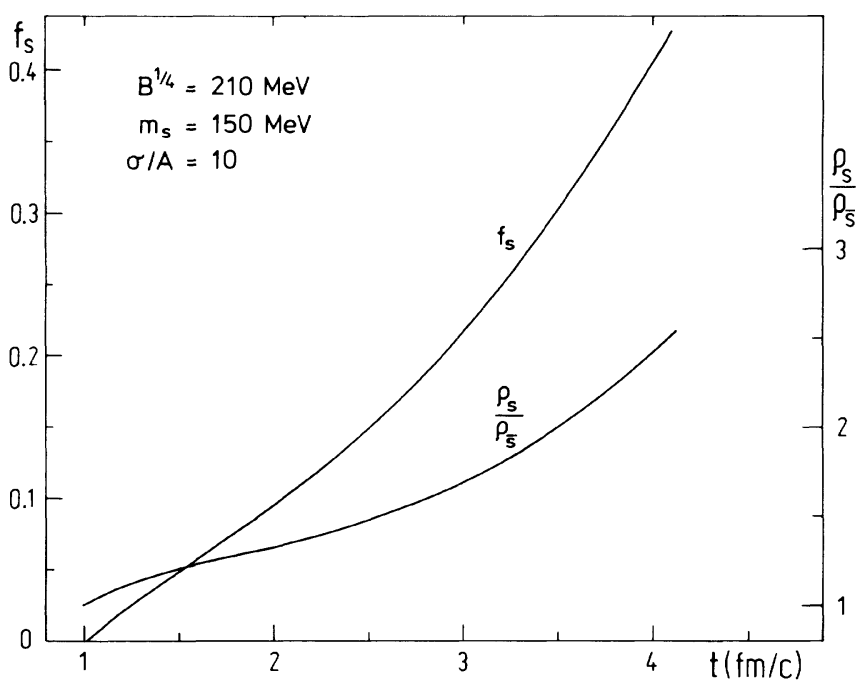

FIG. 2. The time dependence of the $s / \bar{s}$ ratio and of the fraction of strangeness for the isentropic-expansion scenario demonstrated in the previous figures. Note that the strangeness excess becomes largest towards the late stage of the expansion. Rapid expansion can therefore result in the formation of droplets of strange-quark matter, which might be metastable.

must, in addition to the equilibrium expansion used in Refs. 5-7, include the following: In the hadronic sector of the mixed phases the $K^{+}$are enriched and the hyperons are suppressed. The mesons are much lighter than the nucleons and their resonances, their velocity is correspondingly higher, so the rapidly emitted particles, which also cool the system, are mainly the mesons. They carry away entropy, energy, and antistrangeness. Therefore, the whole system is being charged up with strangeness, and this, in turn also enlarges the fraction of strangeness in the remaining quark droplets. Because of this additional increase of the strangeness fraction $f_{s}$, the quark droplets may have a lower energy per baryon than the hadronic phase. This is due to the lowering of the Fermi energy by the increased number of degrees of freedom. Now in an isentropic expansion the system

FIG. 1. The ratio of strange to antistrange quarks in the QGP as a function of the critical baryochemical potential along the phase transition, i.e., for a continuously varying temperature. Observe that in (a), where the original MIT bag-model constant is used, $s / \bar{s}$ exceeds 10 for $\mu_{B}=150 \mathrm{MeV}$. (b) The analogous result obtained with a larger bag constant, $B^{1 / 4}=210 \mathrm{MeV}$. Also regarded is the path which the system must take in an isentropic expansion with $\sigma / A=10$. Note that the ratio does not exceed 3 for this parameter set. (c) The fraction of strange quarks to all quarks present in the QGP as a function of $\mu_{B}$. The same bag-model parameters as in (b) are used. Note that $f_{s}$ can exceed 0.5. The path of an isentropic expansion with $\sigma / A$ is also shown. 
TABLE I. Energy density per baryon in the ground state for strange-quark matter at temperature zero for a given strangeness fraction $f_{s}$, and a certain bag constant and strange-quark mass. Note that we have used $\alpha_{s}=0$. In the last row one finds an estimate of the corresponding hyperon mass with the above strangeness fraction. The underlined numbers indicate stable strange-quark matter with respect to strong decay towards the hadronic hyperon matter.

\begin{tabular}{|c|c|c|c|c|c|c|}
\hline $\begin{array}{c}B^{1 / 4} \\
(\mathrm{MeV})\end{array}$ & $\begin{array}{c}m_{s} \\
(\mathrm{MeV})\end{array}$ & 0.1 & 0.3 & 0.5 & 0.7 & 1 \\
\hline 145 & 150 & $\underline{905}$ & $\underline{887}$ & $\underline{877}$ & $\underline{874}$ & $\underline{879}$ \\
\hline 145 & 279 & $\underline{916}$ & $\overline{919}$ & $\overline{928}$ & $\underline{942}$ & 971 \\
\hline 160 & 150 & 997 & $\underline{975}$ & $\underline{963}$ & $\underline{958}$ & $\underline{962}$ \\
\hline 160 & 279 & 1008 & 1006 & $\underline{1012}$ & 1022 & 1049 \\
\hline 180 & 150 & 1119 & 1094 & $\overline{1077}$ & $\overline{1070}$ & 1072 \\
\hline 180 & 279 & 1131 & 1123 & 1124 & 1131 & 1153 \\
\hline 210 & 150 & 1304 & 1271 & 1250 & 1239 & 1238 \\
\hline 210 & 279 & 1315 & 1299 & 1293 & 1295 & 1313 \\
\hline \multicolumn{2}{|c|}{$f_{s} m_{\Lambda}+\left(1-f_{s}\right) m_{N}$} & 957 & 992 & 1042 & 1063 & 1116 \\
\hline
\end{tabular}

moves towards the energetically most favorable position, which, as was found at zero temperature in Refs. 1-3, is the strangelet. In Table I we show the result for several values of bag constants. Here the ground-state energy per baryon of the quark matter with a given strangeness fraction is compared to a simple estimate of the corresponding mass of hadronic hyperon matter. Note, that for the original MIT bag constant quark matter is stable for all values of $f_{s}$. For the higher values $B^{1 / 4}=160$ and $180 \mathrm{MeV}$, respectively, only quark matter with the net strangeness fraction $f_{s}>0.5$ and $f_{s}>1$, respectively, is lower in energy per baryon than the corresponding hadronic matter. Therefore, once strangeness is built up in the plasma, it can stay in the quark phase. Then it has a chance of cooling down to form metastable cold droplets of strange-quark matter, which can only decay via weak interactions. For large values of $B^{1 / 4}>200 \mathrm{MeV}$, strange-quark matter is not stable. Indeed, for much larger bag constants, $B^{1 / 4}>235 \mathrm{MeV}$, the strangeness separation mechanism proposed here does not work, because associated production would favor the hadronic hyperon matter state and thus strangeness would be enriched in the hadron side.

Some cautionary remarks are in order. In strangelet computations, strange matter is absolutely stable only for large $A>10$. Metastable strangelets could, however, also exist for smaller $A$ values. But we would expect that the probability of forming large plasma droplets in heavy-ion collisions will be small indeed. For a rough es- timate of the production rate of superstrange nuclei we would like to refer to the work of Kerman and Weiss, and Liu and Shaw. ${ }^{16}$

Additional (nonstrange and strange) mesons and baryon resonances should be included into the partition sums. The phase-space factors for these particles can make for large contributions at the energy densities of interest. We would like to emphasize, though, that while our quantitative results may change, the qualitative conclusions remain valid. To improve the model, we will incorporate additional resonances and extend the strangeness calculations to include quantum statistics. Furthermore, a generalized treatment of the phase transition of the QGP to the hadron phase with initial net strangeness not equal to zero is being developed. The nonequilibrium aspects of this transition, meson radiation and quark clustering, need to be studied in the near future. It should help our understanding of the possible formation of strangelets in the deconfinement phase transition, be it in the early Universe or in high-energy heavy-ion collisions.

${ }^{1}$ S. A. Chin and A. K. Kerman, Phys. Rev. Lett. 43, 1292 (1979); J. D. Bjorken and L. D. McLerran, Phys. Rev. D 20, 2353 (1979).

${ }^{2}$ E. Witten, Phys. Rev. D 30, 272 (1984)

${ }^{3}$ E. Farhi and R. L. Jaffe, Phys. Rev. D 30, 2379 (1984).

${ }^{4}$ G. Baym and S. A. Chin, Phys. Lett. 62B, 241 (1976).

${ }^{5}$ P. Koch, B. Mueller, and J. Rafelski, Phys. Rep. 142, 167 (1986); P. Koch, J. Rafelski, and W. Greiner, Phys. Lett. 123B, 151 (1983), and 145B, 142 (1984).

${ }^{6}$ J. Kapusta and A. Mekjian, Phys. Rev. D 33, 1304 (1986).

${ }^{7}$ T. Matsui, B. Svetisky, and L. D. McLerran, Phys. Rev. D 34, 783, 2047 (1986).

${ }^{8}$ K. S. Lee, M. J. Rhoades-Brown, and U. Heinz, Phys. Lett. 174B, 123 (1986).

${ }^{9}$ Quark Matter'83, Nucl. Phys. A418 (1984); Quark Matter 84, Lecture Notes in Physics, Vol. 221, edited by H. Araki, J. Ehlers, K. Hepp, R. Kippenhahn, H. A. Weidenmüller, J. Zittartz, and W. Beiglböck (Springer, Berlin, 1985).

${ }^{10}$ J. Cleymans, R. V. Gavai, and E. Suhonen; Phys. Rep. 130, 217 (1986).

${ }^{11}$ P. Subramanian, H. Stoecker, and W. Greiner, Phys. Lett. 173B, 468 (1986).

${ }^{12}$ U. Heinz, P. R. Subramanian, H. Stoecker, and W. Greiner, J. Phys. G 12, 1237 (1986).

${ }^{13}$ H. Stoecker and W. Greiner, Phys. Rep. 137, 277 (1986).

${ }^{14}$ H. Stoecker, G. Graebner, J. A. Maruhn, and W. Greiner, Phys. Lett. 95B, 192 (1980).

15 J. D. Bjorken, Phys. Rev. D 27, 140 (1983).

${ }^{16}$ A. K. Kerman and M. S. Weiss, Phys. Rev. C 8, 408 (1973); H. Liu and G. L. Shaw, Phys. Rev. D 30, 1137 (1984). 